\title{
Zur relativistischen Quantenmechanik
}

\author{
Von Walter Wessel * \\ (Z. Naturforschg. 4 a, 645-653 [1949]; eingegangen am 17. Mai 1949)
}

\begin{abstract}
Die früher ${ }^{1}$ mitgeteilten Ergebnisse einer Darstellungstheorie der verallgemeinerten Dirac-Gleichung werden nach zwei einander ergänzenden Methoden abgeleitet (Abschnitt 2 und 3). Einzelheiten, auf die wir besonders hinweisen möchten, sind das Verhältnis zu der Theorie von B op p in der Einleitung, die Charakterisierung der Darstellungen durch einen kleinsten Spin im 3. Abschnitt, die Realitätsverhältnisse der endlichen und unendlichen Darstellungen am Ende des 3. Abschnittes und die Auflösung der Wellengleichung nach dem Eigenwertparameter am Schlusse der Arbeit.
\end{abstract}

$\mathrm{I}_{\mathrm{p}}^{\mathrm{n}}$ n mehreren Arbeiten wurde versucht, das Spinphänomen, insofern als darin neue Freiheitsgrade der Elementarteilchen zum Vorschein kommen, aus dem Auftreten höherer Zeitableitungen in ihrer klassischen Bewegungsgleichung zu deuten. Wir gingen dabei zunächst ${ }^{2}$ von punktförmigen Teilchen aus, bei denen die Strahlungskraft Ableitungen bis zur Dritten enthält. Hiermit läßt sich das für das Diracsche Elektron charakteristische Auseinanderfallen von Geschwindigkeit und Impuls verstehen. Um die Bewegungsgleichungen in die Form von Poisson-Klammern zu bringen und damit ihre Quantisierung vorzubereiten, erwies es sich später als notwendig ${ }^{3}$, ein magnetisches Moment der Teilchen als selbständige Variable einzuführen, was dem Auftreten weiterer Zeitableitungen und damit der Annahme einer endlichen Ausdehnung der Teilchen entspricht. Dieses Programm hat neuerdings eine starke Unterstützung erfahren, indem B o p p zeigen konnte ${ }^{4}$, daß endlich ausgedehnte Teilchen schon eine beschleunigungsabhängige Ruhmasse haben, so daß man auch bei Weglassen der eigentlichen Strahlungskraft dritte Ableitungen nach der Zeit erhält. Dies ist eine sehr erwünschte Erkenntnis, denn es war von vornherein klar ${ }^{5}$, daß die Variablen des Spins eine über ihre Verwendung in der Strahlungskraft hinausgehende Bedeutung haben mußten, schon aus dem einfachen Grunde, weil es Elementarteilchen mit Spin, aber

* Z. Zt. in USA.

1 W. Wesse 1, Z. Naturforschg. 3a, 559 [1948]. Im folgenden als 2 zitiert.

${ }_{2}^{2}$ W. W essel, Z. Physik 92, 407 [1934].

3 W. Wessel, Ann. Physik (5) 43, 565 [1943]; Z. Naturforschg. 1, 622 [1946]. Die letztgenannte Arbeit wird im folgenden als 1 zitiert. Sie erschien auch (mit abweichender Formelzählung) als FIAT-Report 1131.

4 F. B op p, Z. Naturforschg. 3a, 564 [1948].

5 W. W essel, Z. Physik 110, 625 [1938]. ohne Ladung, daher ohne Strahlungsreaktion (abgesehen von Momenten höherer Ordnung) gibt.

Bopp konnte die von uns durch eine heuristische Interpretation der Diracschen Algebra aufgestellten Poisson-Klammern folgerichtig aus seiner Lagrange-Funktion ableiten. Hinsichtlich dieser Klammern, d. h. insofern man sie als eine geschlossene Algebra auffaßt, stimmen unsere Formulierungen vollständig überein, wie an Hand der Schlüsseltabelle im nächsten Abschnitt leicht nachzurechnen ist. Ein Unterschied besteht jedoch in der Wahl der zugrundeliegenden kanonischen Variablen. Dieser hat zur Folge, daß eine von den Invarianten des Momententensors bei Bopp verschwindet: die von ihm abgeleiteten $g_{a \rho}$ erfüllen die Identität $g^{a \beta} g_{\alpha \beta}^{*}=0$, wenn $g_{a \beta}^{*}$ den zu $g_{a_{\beta}}$ dualen Tensor bedeutet $(\alpha, \beta=1 \ldots 4)$. In unseren Bezeichnungen bedeutet das $\mathfrak{M} \mathfrak{P}=I J=0$ oder $I=0$, da $J$ nicht mit allen Variablen vertauschbar ist. Die Algebra hat daher bei uns wie bei Dirac 16, bei Bopp nur 15 Elemente. Der Grund für diesen Unterschied ist der, daß bei Bopp nur sechs Variable (die kanonischen $\mathfrak{v}$ und $\mathfrak{g}$ ) unabhängig sind, während sich unsere PoissonKlammern auf sieben unabhängigen Elementen aufbauen ${ }^{6}$. Wir dürfen vielleicht hoffen, daß sich dieser Unterschied noch beheben läßt. Wir betrachten es als einen Vorzug unserer Formulierung, daß dabei die Geschwindigkeitskomponenten, in Analogie zu Dirac, auch in der klassischen

6 Wir gehen von vier komplexen Spinoren aus, d.h. von acht reellen Größen, und bilden daraus die 16 Elemente der Diracschen Algebra. Zwischen diesen bestehen 9 Identitäten, so daß $16-9=7$ Elemente übrigbleiben. Früher wurden versehentlich nur 8 Identitäten aufgezählt [die achte ist die Gl. $(3,26)]$. Hr. K of in k machte mich freundlicherweise darauf aufmerksam, daß nach seiner Aufstellung (Ann. Physik (V) 38, 421 [1940]) diese Abzählung unvollständig ist. In der Tat hat Gl. $(3,26)$ eine duale mit $j M^{*}$ statt $j M$. 


\begin{tabular}{|c|c|c|c|c|c|c|c|c|c|c|c|c|c|}
\hline \multirow[t]{3}{*}{$j_{1}$} & $j_{3}$ & $j_{4}$ & $k_{1}$ & $k_{2}$ & $k_{3}$ & $k_{4}$ & $M_{23}$ & $M_{31}$ & $M_{12}$ & $M_{14}$ & $M_{24}$ & $M_{34}$ & $I$ \\
\hline & $\iota_{3}$ & $\iota_{4}$ & $\%_{1}$ & $\varkappa_{2}$ & $\%_{3}$ & $\varkappa_{4} ;$ & $\mathbf{M}_{1}$ & $\mathrm{M}_{2}$ & $\mathrm{M}_{3}$ & $\Pi_{1}$ & $\Pi_{2}$ & $\Pi_{3}$ & I \\
\hline & $g_{3}$ & $g_{4} ;$ & $g_{51}$ & $g_{52}$ & $g_{53}$ & $g_{54} ;$ & $g_{32}$ & $g_{13}$ & $g_{91}$ & $g_{41}$ & $g_{42}$ & $g_{43}$ & 0 \\
\hline
\end{tabular}

Theorie als nichtvertauschbar im Sinne nichtverschwindender Poisson-Klammern erscheinen [s. Anm. 3, Formeln $(5,11)]$; diese Eigenschaft geht verloren, wenn sie kanonisch sind.

In der vorliegenden Arbeit sollen die PoissonKlammern (P.-K.) als Vertauschungs-Relationen (V.-R.) umgedeutet und Mairixdarstellungen dafür angegeben werden. Es ist für uns das Gegebene, von der 16-gliedrigen Algebra auszugehen und den Fall $I=0$ als Spezialfall aufzufassen. Der große Unterschied der folgenden Darstellungstheorie gegenüber der von $\mathrm{B}$ o $\mathrm{p} \mathrm{p}^{7}$ hat damit nichts zu tun; er baruht vielmehr darauf, daß wir nur hermitesche Matrizen zulassen. Wir kommen damit, wie schon früher ${ }^{1}$ angedeutet, zu durchweg unendlichen Darstellungen. Wenn wir die Argumente noch einmal zusammenstellen dürfen, die uns zugunsten unseres Versuchs zu sprechen scheinen, so sind es die, daß damit alle Matrizen einer unmittelbaren physikalischen Deutung fähig werden und überhaupt, teilweise auch hinsichtlich der Kontinuität ihrer Eigenwerte, auf gleichen Fuß mit Koordinaten und Impulsen kommen. Das Problem der Massenspektren nimmt damit, wie auch schon gezeigt wurde, einen sehr günstigen Aspekt an, und insbesondere erscheint ein unendlich vieldimensionaler Raum der Eigenvektoren als das geeignete Substrat zur Einordnung der Strahlungskraft, die nach wie vor unser Ziel bleibt. Auch Hill $1^{8}$ erkannte bereits die Notwendigkeit kontinuierlicher Operatoren in der Dirac-Gleichung. Im Gegensatz zu den Arbeiten vieler anderer Autoren kennen unsere Darstellungen keine Klassifizierung nach einem bestimmten Spin, sondern nur nach einem kleinsten Spin. Es ist aber anzunehmen, daß die Teilchen in niederen Näherungen wie Teilchen mit diesem Spin reagieren.

1. Umdeutung der Poisson-Klammern. Nomenklatur

Soweit die P.-K. von 1 auf Produkte vertauschbarer Faktoren führen, können sie ohne weiteres in V.-R. umgedeutet werden, indem man rechterhand, unter Einführung einer Konstanten von der Dimen-

7 F. B ор u. F. L. B a u er, diese Z. 4a, 611 [1949].

8 E. L. Hill, Physic. Rev. (2) 73, 910 [1948]. sion einer Wirkung, einen Faktor $i=V-1$ einfügt. Wir denken uns wieder wie früher ${ }^{1}$ alle dimensionierten Größen (lateinische Buchstaben) mit Hilfe der Konstanten $\Gamma$ und $\Lambda$ von 1 als Vielfache dimensionsloser (griechische Buchstaben) ausgedrückt. Die Übersetzung vermittelt obenstehende Tabelle. In der dritten Zeile sind die entsprechenden Größen von B op p angefügt.

Unsere $u_{k}$ sind die $j_{k} / \sqrt{I^{2}+J^{2}}$, das sind die $\boldsymbol{u}_{k} / \boldsymbol{c}=g_{k} / g_{5}$ bei Bopp; unsere $U_{k}$ entsprechen seinen $g_{5 k} / g_{5}$. Alle Komponenten sind reell; Ziehen des Index 4 verkehrt das Zeichen.

Zur Umdeutung geeignet sind nun entweder die Spinor-Relationen $(1,4,10)$ oder die Relationen $(4,13)$, $(4,16)-(4,22)$ der Tensoren, aus denen die Spinoren herausfallen. Es könnte wohl sein (ein Teil unserer Betrachtungen in 2 beruhte auf der Unterstellung dieser Möglichkeit), daß die Tensor-Relationen mehr Darstellungen haben als die Spinor-Relationen, aus denen sie hervorgehen, ebenso wie bekanntlich die V.-R. des Drehimpulses halbzahlige Darstellungen haben, die sich mit Koordinaten und Impulsen nicht erfüllen lassen. Die in der vorliegenden Arbeit gegebenen Darstellungen, auch die halbzahligen, sind jedoch beiden gemeinsam.

Im folgenden werden wir hauptsächlich das von den Tensor-Relationen ausgehende Verfahren beschreiben, weil es unter Benutzung in der Literatur bereits vorhandener Analysen die Darstellungen in geschlossener und vollständig ausreduzierter Form liefert. Wegen des Zusammenhanges mit den ursprünglichen P.-K. muß jedoch auch das von den Spinoren ausgehende Verfahren erläutert werden. Das kann um so leichter geschehen, als' es ganz automatisch abläuft ${ }^{9}$. Es hat übrigens den Vorzug, die Eigenwertspektren der wichtigsten Darstellungselemente I und $\mathbf{a}^{4}$ in sehr übersichtlicher Form zu liefern; auch sind die Beziehungen $(3,21)$ ff., die wir hier nur verifizieren, nicht wohl zu erraten, wenn man sich nicht einige Schritte der dahin führenden unitären Transformation ausrechnet.

\section{Darstellungen I. Art: Spinor-Relationen}

Wir nehmen also wie in Gl. (7) von 2 an:

$$
\begin{aligned}
{\left[\psi_{\mu} \chi^{v^{\prime}}\right] } & =\delta_{\mu}^{v} & {\left[\psi_{\dot{\mu}} \chi^{\dot{v}}\right] } & =-\delta_{\dot{\mu}}^{\dot{ }}, \\
\mu, \nu & =1,2 & \dot{\mu}, \dot{v} & =\mathrm{j}, \dot{2}
\end{aligned}
$$

sämtliche übrigen Klammern Null. Die LorentzInvarianz dieser Beziehungen ist für die P.-K.

• Eine ausführliche Darstellung ist im Druck bei der Physical Review. (Erscheint voraussichtlich in Heft 10 des Bd. 76 [1949].) 
in 1 bewiesen und überträgt sich ohne weiteres auf die V.-R. Um auf kanonische Variable im gewöhnlichen Sinne zu kommen, braucht man nur die Real- und Imaginärteile dieser Spinoren in der Form

$$
\begin{array}{ll}
p_{1}=\frac{1}{\sqrt{2}}\left(\psi_{1}+\psi_{\mathrm{i}}\right) & q_{1}=\frac{1}{i \sqrt{2}}\left(\chi^{1}-\chi^{\mathrm{i}}\right) \\
p_{2}=\frac{1}{i \sqrt{2}}\left(\psi_{1}-\psi_{\mathrm{i}}\right) & q_{2}=\frac{1}{\sqrt{2}}\left(\chi^{1}+\chi^{\mathrm{i}}\right)
\end{array}
$$

einzuführen. Diese Verbindungen erfüllen in der Tat, wie man leicht nachrechnet, die Relationen

$$
\left[p_{m} q_{n}\right\rfloor=\frac{1}{i} \delta_{m n}, \quad m, n=1,2 .
$$

Verfährt man mit den Spinoren des Index 2 ebenso, so erhält man zwei weitere unabhängige Paare $p_{3}, p_{4}, q_{3}, q_{4}$, womit sich die Gültigkeit von $(2,3)$ auf $m, n=1 \ldots 4$ erhöht. Diese Pseudokoordinaten und -Impulse haben natürlich keine einfachen Transformationseigenschaften (sind weder Vierervektoren noch Spinoren), und es ist recht merkwürdig, daß man mit ihnen Relationen der Form $(2,3)$ erfüllen kánn. Die Lorentz-Invarianz unseres Verfahrens beruht zunächst auf $(2,1)$ und folgt weiter unten direkt aus dem im Anschluß an $(3,3)$ und $(3,4)$ Gesagten.

Drückt man nun unsere wie früher [in 1, Formeln $(3,11)$ und $(3,7)]$ in der Notation von Laporte und Uhlenbeck ${ }^{\mathbf{1 0}}$ geschriebenen $M_{i k}$. in diesen Variablen aus, so erscheinen sie jeweils zusammengesetzt aus zwei Ausdrücken in der Form von Drehimpulskomponenten. Eine kanomische Transformation $p_{k}=\partial S / \partial q_{k}, \vec{q}_{k}=\partial S / \partial \vec{p}_{k}$ mit der Erzeugenden

$$
\begin{aligned}
& S\left(q_{1} \cdots q_{4} ; \bar{p}_{1} \cdot \bar{p}_{4}\right) \\
& \quad=\sqrt{2} \sum_{k=1}^{4} \bar{p}_{k} q_{k}-\bar{p}_{1} \bar{p}_{2}-\bar{p}_{3} \bar{p}_{4}-q_{1} q_{2}-q_{3} q_{4}
\end{aligned}
$$

bringt dann die vier Matrizen $\mathrm{I}, \imath^{4}, \mathrm{M}_{3}, x_{3}$ in die Form

$$
\begin{aligned}
\mathrm{M}_{3} & =1 / 2\left(-H_{1}+H_{2}+H_{3}-H_{4}\right) \\
\mathrm{I} & =1 / 2\left(H_{1}-H_{2}+H_{3}-H_{4}\right) \\
\varkappa_{3} & =1 / 2\left(H_{1}+H_{2}-H_{3}-H_{4}\right) \\
\iota^{4} & =1 / 2\left(H_{1}+H_{2}+H_{3}+H_{4}\right),
\end{aligned}
$$

10 O. Laporte u. G. E. Uhlenbeck, Physic. Rev. 37, 1380 u. 1552 [1931]. wobei die $H_{k}$ die Bedeutung

$$
H_{k}=1 / 2\left(p_{k}^{2}+\bar{q}_{k}^{2}\right),
$$

also die Eigenwerte $n_{k}+1 / 2, n_{k}=0,1,2,3 \ldots$ haben. Die vier Größen unter $(2,5)$ sind also sämtlich ganzzahlig oder halbganz, und mit ihren Eigenwerten kann man die übrige Darstellung beziffern. Die Wahl des Wertes von I, ganz oder halbganz, bestimmt jeweils eine von untereinander nicht kombinierenden Klassen, da nach 1 $(4,19)$ die Invariante I mit allen andern Variablen vertauschbar ist.

Innerhalb jeder Klasse ist das natürliche Ausgangselement $~_{4}^{4}$. Es ist positiv definit; sein niedrigster Wert ist wegen der "Nullpunkts"-Terme in $(2,6)$ Eins. Jeder andere Wert kann nur durch eine endliche Anzahl von Kombinationen $n_{1} \ldots n_{4}$ hergestellt werden. Bei gegebenem $I$ hat man nach $(2,5) \quad:^{4}=n_{2}+n_{4}+\mathrm{I}+1=n_{1}+n_{3}-\mathrm{I}+1$, d. h. immer

$$
\iota^{4} \geqslant|\mathrm{I}|+1
$$

Jeder $\imath^{4}$-Wert ist noch $\left(\imath^{4}-\mathrm{I}\right)\left(\mathrm{\imath}^{4}+\mathrm{I}\right)$-fach entartet, denn es können noch $:^{4}-\mathrm{I}$ Kombinationen $n_{2}+n_{4}$ mit $\iota^{4}+$ I Kombinationen $n_{1}+n_{3}$ vereinigt werden. Da $\mathbf{M}_{1}, \mathbf{M}_{2}, \mathbf{M}_{3}$ und $x_{1}, x_{2}, x_{3}$ mit $\imath^{4}$ vertauschbar sind, zerfallen sie in quadratische Stufenmatrizen mit dieser Zeilen- und Kolonnenzahl. Damit hat man alle in 2 behaupteten Ergebnisse bis auf die kontinuierlichen Spektren, die sich übersichtlicher aus der Tensor-Darstellung ergeben.

Der Nachteil der eben ausgeführten Darstellung ist der, daß sich das Bildungsgesetz der Matrizen nicht sehr übersichtlich schreiben läßt, und daß die eben genannten Stufenmatrizen nicht in voll reduzierter Form erscheinen. Man kann sie noch nach den Darstellungen der Drehungsgruppe weiter zerlegen. Um sie in dieser Normalform zu erhalten, geht man besser von den Tensor-Relationen aus.

\section{Darstellungen II. Art:}

Tensor-Relationen

Die darzustellenden Beziehungen lassen sich übersichtlich nach Art einer Gruppentafel zusammenfassen, wenn man darin die Reihen durch den Faktor $a$, die Spalten durch den Faktor $b$ in der Relation $[a b]=a b-b a$ bezeichnet und an ihrer Kreuzung $1 / i[a b]$ einträgt. Die später benutzten $\mathbf{M}_{k}, \Pi_{k}$ sind hier noch als $\mathbf{M}_{i k}$ bezeichnet. 


\begin{tabular}{c|c|c|c|c|c|}
\multicolumn{2}{c}{$\mathrm{I}$} & $\mathrm{K}$ & $\iota^{l}$ & $\varkappa^{l}$ & $\mathrm{M}^{r s}$ \\
$\mathrm{I}$ & 0 & 0 & 0 & 0 & 0 \\
\hline $\mathrm{K}$ & 0 & 0 & $\varkappa^{l}$ & $\iota^{l}$ & 0 \\
\hline$\iota_{i}$ & 0 & $-\varkappa_{i}$ & $-\mathrm{M}_{i}^{l}$ & $-\mathrm{K} \delta_{i}^{i}$ & $\delta_{i}^{s} \iota^{r}-\delta_{i}^{r} \iota^{s}$ \\
\hline$\varkappa_{i}$ & 0 & $-\iota_{i}$ & $\mathrm{~K} \delta_{i}^{l}$ & $\mathrm{M}_{i}^{l} \cdot$ & $\delta_{i}^{s} \varkappa^{r}-\delta_{i}^{r} \varkappa^{s}$ \\
\hline $\mathrm{M}_{i k}$ & 0 & 0 & $\delta_{i}^{l} \iota_{k}-\delta_{k}^{l} \iota_{i}$ & $\delta_{i}^{l} \varkappa_{k}-\delta_{k}^{l} \varkappa_{i}$ & $\delta_{i}^{r} \mathrm{M}_{k}^{s}-\delta_{k}^{r} \mathrm{M}_{i}^{s}$ \\
\hline & & & & & $-\delta_{i}^{s} \mathrm{M}_{k}^{r}+\delta_{k}^{s} \mathrm{M}_{i}^{r}$ \\
\hline
\end{tabular}

Von den Nebenbedingungen, die diese Größen in 1 noch erfüllen, wird für die Bestimmung der Darstellungen nur $(3,24)$ in 1 gebraucht. In unseren jetzigen Bezeichnungen und im Operatorsinne lauten sie

$$
\begin{aligned}
\overrightarrow{\mathrm{M}}^{2}-\vec{\Pi}^{2} & =\mathrm{I}^{2}-\mathrm{K}^{2}-1 \\
\overrightarrow{\mathrm{M}} \vec{\Pi} & =\mathrm{IK} .
\end{aligned}
$$

Der Zusatz - 1 ergibt sich aus der Darstellung in Abschn. 2; das Minuszeichen in der zweiten Gleichung in 1 war irrtümlich.

Wir beginnen mit der Darstellung der $M_{k}$ bzw. $M_{k}, \Pi_{k}$. Ihre $\mathrm{V}$.-R. finden sich in der rechten unteren Ecke von $(3,1)$. Man betrachtet sie am besten in der Reihenfolge

$$
\begin{aligned}
{\left[\mathrm{M}_{i} \mathrm{M}_{k}\right] } & =i \mathrm{M}_{l} \\
{\left[\mathrm{M}_{i} \Pi_{k}\right] } & =i \Pi_{l} \\
{\left[\Pi_{i} \Pi_{k}\right] } & =-i \mathrm{M}_{l} \\
(i k l) & =(123)
\end{aligned}
$$

und

$$
\left[\mathrm{M}_{1} \Pi_{1}\right]=\left[\mathrm{M}_{2} \Pi_{2}\right]=\left[\mathrm{M}_{3} \Pi_{3}\right]=0 .
$$

Diese Relationen machen die M , $\Pi_{k}$ zu dem „Kern“ der Darstellungen der Lorentz-Transformation ${ }^{11}$. In der Form

$$
U_{k}=\exp i \varphi\left\{\begin{array}{l}
\mathbf{M}_{k} \\
\Pi_{k}
\end{array}\right.
$$

11 B. L. van der Waerden, Die gruppentheoretische Methode in der Quantenmechanik, Berlin 1932.

12 V.Bargmann, Ann. of Math. (2) 48, 568 [1947]; $\mathrm{H}$ a r is h-Chandra, Proc. Roy. Soc. [London], Ser. A 189, 372 [1947]; I. Gelf and u. M. Neumark, Bull. Acad. Sci. URSS, Sér. physique 10, 93 [1946]. bilden sie die unitären Matrizen, die Drehungen $\left(\mathbf{M}_{k}\right)$ um einen Winkel $\varphi$ bzw. Lorentz-Transformationen $\left(\Pi_{k}\right)$ aller übrigen Größen des Schemas $(3,1)$ vermitteln, wie man ausgehend von infinitesimalen Transformationen leicht nachweist.

Diese unitären Darstellungen sind in den letzten Jahren von mehreren Autoren eingehend studiert worden ${ }^{12}$. Wigne ${ }^{13}$ erkannte schon vor langem, daß sie unendlich sind. Wir können unmittelbar an $\mathrm{H}$ arish-Chandra anknüpfen. Die Aufgabe ist, zu den sechs $\mathbf{M}_{k}, \Pi_{k}$-Matrizen, die in dem Schema $(3,1)$ die Natur einer Untergruppe (Subalgebra) haben, die $x_{k}$ - und besonders die $k_{k}^{-}$ Matrizen hinzuzukonstruieren, die die Wellengleichung aufbauen.

Das erste Tripel $(3,3)$ bildet einfach die V.-R. des Drehimpulses; man hat also mit

$$
\mathrm{M}_{1}+i \mathrm{M}_{2}=\mathrm{M}, \quad \mathrm{M}_{1}-i \mathrm{M}_{2}=\mathrm{M}^{\dagger}
$$

(† transformiert und konjugiert) einfach $\left(\varkappa \mu|\mathbf{M}| \boldsymbol{\varkappa}^{\prime} \mu^{\prime}\right)$

$$
\begin{aligned}
& =\sqrt{(x+\mu)(x-\mu+1)} \delta_{\varkappa \varkappa^{\prime}} \delta_{\mu, \mu^{\prime}+1} \\
& \left(x \mu\left|\mathrm{M}_{3}\right| \varkappa^{\prime} \mu^{\prime}\right)=\mu \cdot \delta_{\varkappa \varkappa^{\prime}} \delta_{\mu \mu^{\prime}} \quad(3,7) \\
& x \text { ganz oder halbganz }(\geqslant 0), \\
& \quad x \geqslant \mu \geqslant-\varkappa .
\end{aligned}
$$

Für die $\mathrm{II}_{k}$ setzen wir mit $\mathrm{Harish}-\mathrm{Ch}$ andra ${ }^{15}$ nach Weyl ${ }^{14}$

$$
\Pi_{1}+i \Pi_{2}=\Pi, \quad \Pi_{1}-i \Pi_{2}=\Pi^{\dagger}
$$

13 E. Wi gne r, Ann. of Math. 40, 149 [1939].

${ }_{14} \mathrm{H}$. We y l, Gruppentheorie und Quantenmechanik, Leipzig 1931. 
$\left(\varkappa \mu|\Pi| \varkappa^{\prime} \mu^{\prime}\right)$

$$
\begin{aligned}
&=\left\{-a_{\varkappa} \sqrt{(\varkappa+\mu)(\varkappa+\mu-1)} \delta_{\varkappa, \varkappa^{\prime}+1}\right. \\
&+b_{\varkappa} \sqrt{(\varkappa+\mu)(\varkappa-\mu+1)} \delta_{\varkappa \varkappa^{\prime}} \\
&+\left.a_{\varkappa+1}^{\dagger} \sqrt{(\varkappa-\mu+1)(\varkappa-\mu+2)} \delta_{\varkappa, \varkappa^{\prime}-1}\right\} \delta_{\mu, \mu^{\prime}+1} . \\
&\left(\varkappa \mu\left|\Pi_{3}\right| \varkappa^{\prime} \mu^{\prime}\right) \\
&=\left\{a_{\varkappa} \sqrt{(\varkappa+\mu)(\varkappa-\mu)} \delta_{\varkappa, \varkappa^{\prime}+1}\right. \\
& \quad+b_{\varkappa} \cdot \mu \cdot \delta_{\varkappa \varkappa^{\prime}} \\
&\left.+a_{\varkappa+1}^{\dagger} \sqrt{(\varkappa+\mu+1)(\varkappa-\mu+1)} \delta_{\varkappa, \varkappa^{\prime}-1}\right\} \delta_{\mu, \mu^{\prime}} .
\end{aligned}
$$

Unter $a_{\varkappa}^{\dagger}$ ist zunächst einfach die zu $a_{\varkappa}$ konjugiert komplexe Zahl zu verstehen; $b$ kann als reell angenommen werden. Da wir die $a, a^{\dagger}, b$ weiter unten als Matrizen zu betrachten haben, achten wir in Produkten auf die Faktorenfolge. Diese $\Pi$-Matrizen erfüllen $(3,4)$ und das zweite Tripel $(3,3)$ ohne weitere Voraussetzungen über die $a_{\varkappa}$ und $b_{\%}$.

Bedingungsgleichungen für diese Größen ergeben sich, wenn man in das dritte Tripel $(3,3)$ einsetzt, weil je zwei $\Pi_{k}$ ein von $a_{\varkappa}$ und $b_{\varkappa}$ freies $\mathbf{M}_{k}$ ergeben müssen. Man findet nach einiger Rechnung

$$
\begin{gathered}
(\varkappa-1) a_{\varkappa} b_{\varkappa-1}=(\varkappa+1) b_{\varkappa} a_{\varkappa} \\
(\varkappa-1) b_{\varkappa-1} a_{\varkappa}^{\dagger}=(\varkappa+1) a_{\varkappa}^{\dagger} b_{\varkappa} \quad(3,10) \\
(2 \varkappa+3) a_{\varkappa+1}^{\dagger} a_{\varkappa+1}-(2 \varkappa-1) a_{\varkappa} a_{\varkappa}^{\dagger}-b_{\varkappa}^{2}=1 .
\end{gathered}
$$

$\mathrm{Zu}$ ihrer Auflösung ziehen wir nun die $\mathrm{I}, \mathrm{K}$ von Gl. $(3,2)$ heran. Diese Invarianten spielen im Schema der V.-R. (3,3) dieselbe Rolle wie das drehinvariante Quadrat des Impulsmoments in der Quantentheorie des Drehimpulses. Sie sind nach $(3,1)$ mit allen $\mathbf{M}_{k}$ und $\Pi_{k}$ vertauschbar und können daher, solange man im Rahmen dieser

15 Die Buchstaben $a$ und $b$ an Stelle der $p$ bei HarishChandra sind gewählt, um Verwechslung von + und $\dagger$ $\mathrm{zu}$ vermeiden. Zur Ubersetzung der übrigen Bezeichnungen diene die folgende Tabelle:

$$
\begin{array}{lcccccc}
\mathrm{HCh} & I & J^{2} & k & m & j & \mu \\
\text { wir } & \mathrm{IK} & \mathrm{I}^{2}-\mathrm{K}^{2}-1 & \varkappa & \mu & \mathrm{I} & \mathrm{K} .
\end{array}
$$

Wesentlich ist die Zerlegung von $I$ in die zwei Faktoren I und $\mathbf{K}$. Bei $\mathrm{HCh}$, der sich nur mit den $\mathbf{M}_{k}, \Pi_{k}$ befaßt, sind $I$ und $J^{2}$ mit allen Größen vertauschbar. In unserem Schema $(3,1)$ trifft das nur für I, nicht für K zu. Die Größe $:^{4}$, die bei uns neben I, $x$ und $\mu$ das vierte klassifizierende Element ist, kommt bei $\mathrm{HCh}$ nicht vor.
Größen bleibt, als $c$-Zahlen behandelt werden. Jede Lorentz-Darstellung wird daher durch zwei Parameter charakterisiert. Man erhält nun aus der zweiten Relation $(3,2)$ einfach

$$
b_{\varkappa}=\frac{\mathrm{IK}}{\varkappa(\varkappa+1)} .
$$

Das ist Gl. (18) bei Harish-Chandra. Sie führt die $b_{\varkappa}$ unmittelbar auf die beiden Invarianten zurück. Die erste Relation ergibt ähnlich

$-(\varkappa+1)(2 \varkappa+3) a_{\%+1}^{\dagger} a_{\varkappa+1}-\varkappa(2 \varkappa-1) a_{\varkappa} a_{\%}^{\dagger}$

$+\varkappa(\varkappa+1)\left(1-b_{\varkappa}^{2}\right)=\mathrm{I}^{2}-\mathrm{K}^{2}-1$.

Durch Verbindung dieser Gleichung mit der dritten Gl. $(3,10)$ ergibt sich sofort, mit Benutzung von $(3,11)$ :

$$
\begin{aligned}
a_{\varkappa} a_{\varkappa}^{\dagger} & =\left(1-\frac{\mathrm{I}^{2}}{\varkappa^{2}}\right) \mathrm{K}_{\varkappa}^{2}+\varkappa^{2} \\
a_{\varkappa+1}^{\dagger} a_{\varkappa+1} & =\left(1-\frac{\mathrm{I}^{2}}{(2 x-1)(2 \varkappa+1)}\right) \frac{\mathrm{K}_{\varkappa}^{2}+(\varkappa+1)^{2}}{(2 \varkappa+1)(2 \varkappa+3)} .
\end{aligned}
$$

Sieht man hier von dem Index $x$ an $\mathrm{K}$ ab, den wir mit Hinblick auf das folgende hinzugefügt haben, so sind die beiden Formeln $(3,13)$ gleichbedeutend unter sich und mit Formel (16) bei Harish Chandra. (Man beachte $j=\mathrm{I}, I / j=\mathrm{K}$.) Damit die $\Pi_{k}$-Matrizen hermitesch reell sind (die Darstellung unitär ist), muß $a a^{\dagger} \geqslant 0$, also

$$
\mathrm{I}^{2}=x_{\min }^{2}
$$

sein, in Worten: der Betrag von I ist der kleinste in der Darstellung vorkommende Wert von $x$. Die beiden ersten Formeln $(3,10)$ sind, da wir die Faktoren noch als vertauschbar betrachten, schon durch $(3,11)$ erfiillt.

Der Wert von I tritt damit in diesen unendlichen Darstellungen an die Stelle eines gegebenen Spins der Teilchen ${ }^{16}$. Nach dem in 1 Gl. $(6,22)$ bewiesenen Erhaltungssatze stellt ja die Matrix $-\mathfrak{M} / \Gamma \Lambda$ den Spin dar. Unser $x$ ist der Maximalwert der Komponenten von $\vec{M}$, also in der Form $\sqrt{x(x+1)}$ der Absolutwert des Spins. Die $x$-Werte sind in unseren Darstellungen nicht beschränkt, vielmehr

16 Die folgende Auffassung ist offenbar im Rahmen unserer Theorie natürlicher als der Begriff des ,reinen“ Spins, zu dem Harish-Chandra auf Grund der von ihm angenommenen Form der Wellengleichung geführt wird. 
zerfällt jede $\mathbf{M}_{k}$-Matrix in Stufenmatrizen [die in $(3,28)$ leeren Diagonalfelder] von beständig zunehmendem Rang. Charakteristisch ist nur der kleinste Spin, weil er wegen $(3,14)$ die Invariante I und damit eine nichtkombinierende Darstellungsklasse bestimmt. Man kann wohl erwarten, daß die Teilchen in niederen Näherungsstufen immer mit diesem Spin in Erscheinung treten und daß höhere Werte nur für feinere Effekte oder unter außergewöhnlichen Bedingungen eine Rolle spielen.

Um weiterzukommen, dürfen wir nun $\mathrm{K}$ bzw. $b$ [vgl. $(3,11)]$ nicht länger als $c$-Zahlen betrachten, denn $\mathrm{K}$ ist $\mathrm{zwar}$ mit allen $\mathrm{M}_{k}, \Pi_{k}$, aber nicht mit den ${ }_{k}$ und $x_{k}$ vertauschbar. Diese Invariante bildet vielmehr mit $:^{4}$ und $x^{4}$ den Zykhs

$\left[\mathrm{K} \iota^{4}\right]=i x^{4},\left[\iota^{4} x^{4}\right]=i \mathrm{~K},\left[x^{4} \mathrm{~K}\right]=-i \iota^{4}$,

der die Grundlage unserer Betrachtungen in 2 war. Wie dort schon ausgeführt, sind die Eigenwerte von : $^{4}$ diskret mit Abstand 1, die von $\mathrm{K}$ und $x^{4}$ kontinuierlich. Wir werden daher wieder neben $\mu$ und seiner oberen Grenze $x$ die Eigenwerte von ${ }^{4}$ zur Bezifferung heranziehen. (In dieser Verwendung möge der Index 4 aus typographischen Gründen gelegentlich wegbleiben.)

Aus $(3,15)$ ergibt sich mit der üblichen Schlußweise (durch Einführung von $\mathrm{K}+i \varkappa^{4}$ und $\left.\mathrm{K}-i x^{4}\right)$, daß $\mathrm{K}$ und $x^{4}$ die Form

$$
\begin{aligned}
& \left(\iota^{4}|\mathrm{~K}| \iota^{\iota^{*}}\right)=A_{\iota} \delta_{\iota, \iota^{\prime}+1}+A_{\iota^{\prime}}^{*} \delta_{\iota, \iota^{\prime}-1} \\
& \left(\iota^{4}\left|\chi^{4}\right| \iota^{4^{\prime}}\right)=i A_{\iota} \delta_{\iota, \iota^{\prime}+1}-i A_{\iota^{\prime}}^{*} \delta_{\iota, \iota^{\prime}-1}
\end{aligned}
$$

haben müssen ( $A_{\iota}$ und $A_{\iota}^{*}$ komplex konjugiert). Die letzte Gl. $(3,15)$ verlangt dann

$$
\left|A_{\iota+1}\right|^{2}-\left|A_{\iota}\right|^{2}=1 / 2 \iota^{4} .
$$

Der Vertauschbarkeit der Drehimpulskomponenten mit ihrer Quadratsumme entspricht hier die Vertauschbarkeit von $\mathrm{K}, x^{4}$ und $\iota^{4}$ mit

$$
Q^{2}=\left(\boldsymbol{x}^{4}\right)^{2}+\mathrm{K}^{2}-\left(\iota^{4}\right)^{2} .
$$

Durch Einsetzen von $(3,16)$ ergibt sich daraus

$$
\left|A_{\iota+1}\right|^{2}+\left|A_{\iota}\right|^{2}=1 / 2\left\{\varrho^{2}+\left(\iota^{4}\right)^{2}\right\},
$$

und daraus folgt in Verbindung mit $(3,17)$

$$
\left|A_{\iota}\right|^{2}=1 / 4\left\{\varrho^{2}+\iota^{4}\left(\iota^{4}-1\right)\right\} .
$$

Wir zeigen nun, daß sich die gesuchte Normalform unserer Darstellung von Abschn. 2 ergibt, wenn man

$$
Q^{2}=-\varkappa(\varkappa+1)
$$

setzt. Damit wird zunächst nach $(3,16)$, unter leichter Umformung der $A_{\ell}$ :

$$
\begin{aligned}
\left(\iota^{4} \mid \mathrm{K} \iota^{4^{\prime}}\right) & =1 / 2\left\{\sqrt{\left(\iota^{4}+x\right)\left(\iota^{4}-x-1\right)} \delta_{\iota, \iota^{\prime}+1}\right. \\
+ & \left.\sqrt{\left(\iota^{4}+x+1\right)\left(\iota^{4}-x\right)} \delta_{\iota, \imath-1}\right\} \cdot(3,
\end{aligned}
$$

Trägt man dies in $(3,13)$ ein, so werden wegen der x-Abhängigkeit von $\mathrm{K}$ die beiden Gleichungen verschieden. Sie müssen dann als Matrizengleichungen verstanden werden, was wir durch Beachtung der Faktorenfolge bei ihrer Ableitung schon vorbereitet haben. Es ist nun nicht allzu schwer zu finden, daß sich die $a_{\%}, a_{\%}^{\dagger}$ mit dem gemeinsamen Faktor

durch

$$
c_{\%}=\frac{ \pm \sqrt{1-\mathrm{I}^{2} / \varkappa^{2}}}{\sqrt{(2 \varkappa-1)(2 \varkappa+1)}}
$$

$$
\begin{aligned}
& \left(\iota^{4}\left|a_{\varkappa}\right| \iota^{4^{\prime}}\right) \\
& =1 / 2 c_{\varkappa}\left\{\sqrt{\left(\iota^{4}+\varkappa\right)\left(\iota^{4}+x-1\right)} \delta_{\iota, \iota^{\prime}+1}\right. \\
& \left.\quad+\sqrt{\left(\iota^{4}-\varkappa\right)\left(\iota^{4}-\varkappa+1\right)} \delta_{\iota, \iota^{\prime}-1}\right\} \\
& \left(\iota^{4}\left|a_{\varkappa}^{\dagger}\right| \iota^{4 \prime}\right) \\
& =1 / 2 c_{\varkappa}\left\{\sqrt{\left(\iota^{4}-x-1\right)\left(\iota^{4}-x\right)} \delta_{\iota, \iota^{\prime}+1}\right. \\
& \left.\quad+\sqrt{\left(\iota^{4}+x+1\right)\left(\iota^{4}+x\right)} \delta_{\iota, \iota^{\prime}-1}\right\}
\end{aligned}
$$

darstellen lassen. Diese Ausdrücke erfüllen ebenfalls die beiden ersten Formeln $(3,10)$.

Hiermit ist alles gegeben: die $\mathbf{M}_{1}, \mathbf{M}_{2}, \mathbf{M}_{3}$. entstehen aus $(3,7)$ durch bloße Ergänzung eines Faktors $\delta_{\iota \iota^{\prime}}$, die $\Pi_{1}, \Pi_{2}, \Pi_{3}$ aus $(3,8),(3,9)$ durch Einsetzen von $(3,11)$ und der eben abgeleiteten Ausdrücke $(3,23)$ und $(3,24)$. Durch Vertauschen mit der Diagonalmatrix $:^{4}$ erhält man nach dem allgemeinen Schema $(3,1)$ sofort die $\iota_{k}$-Komponenten in der Form

$$
\begin{aligned}
\iota_{k} & =i\left[\iota^{\mathbf{4}} \Pi_{k}\right] \\
k & =1,2,3,
\end{aligned}
$$

und durch Vertauschen von K, Gl. $(3,22)$, mit :4 entsteht $x^{4}$, das dann ebenso

$$
\begin{aligned}
\varkappa_{k} & =i\left[\varkappa^{4} \Pi_{k}\right] . \\
k & =1,2,3
\end{aligned}
$$

erzeugt.

Die Aquivalenz der vorliegenden Darstellung 
mit der von Abschn. 2 ist wohl schon ziemlich deutlich. Wir haben erstens gleiche Eigenwerte der $\mathbf{I}$, denn da $\mathrm{I}^{2}$ gleich dem kleinsten Eigenwerte von $x^{2}$ ist und $x$ von Hause aus alle positiven ganzen und halbganzen Zahlen durchlaufen kann, ist die betreffende Formel unter $(2,5)$ bestätigt. Nach $(3,22)$ oder $(3,24)$ beginnt $~^{4}$ mit dem kleinsten Werte von $x+1$; das ist die Aussage von $(2,7)$. Umgekehrt läuft $x$ bei gegebenem $:^{4}$ bis $:^{4}-1$, im ganzen also von $|\mathrm{I}|$ bis $:^{4}-1$. Da jeder dieser Werte noch $(2 x+1)$-fach entartet ist, gehören also zu jedem ${ }^{4}$-Werte noch Teilmatrizen vom Range $2|\mathrm{I}|+1,2|\mathrm{I}|+3, \ldots, 2:^{4}-1$, und die Gesamtheit dieser Stufen ist wieder $\left(\imath^{4}-\mathrm{I}\right)$ $\cdot\left(\imath^{4}+\mathrm{I}\right)$ wie in Abschn. 2.

Die Darstellungen lassen sich also unitär ineinander transformieren. Daraus folgt, da die Matrizen des zweiten Abschnittes alle Relationen $(3,1)$ nach Konstruktion erfüllen, daß die nach $(3,25)$ und $(3,26)$ gebildeten ${ }_{k}$ und $x_{k}$ auch die noch übrigen Relationen, z. B.

$$
\left[\iota_{i} \iota_{k}\right]=-i \mathrm{M}_{i k}
$$

erfüllen, mit denen sich die Algebra schließt.

Als Beispiel für den allgemeinen Aufbau folge hier $2 \mathrm{~K}$ für die Darstellung mit $\mathrm{I}=1 / 2$ :

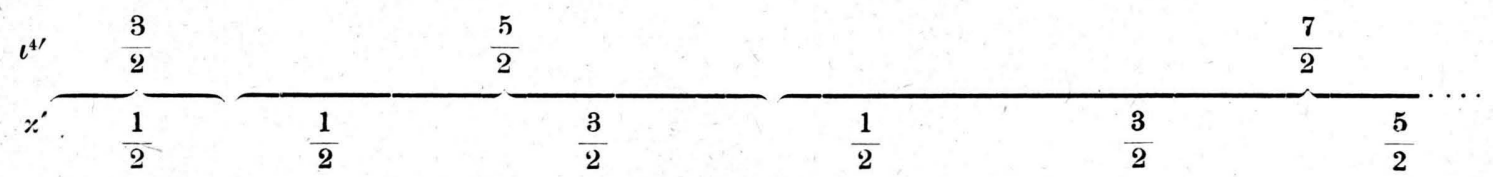

$\iota^{4} \% \quad \mu \frac{1}{2} \frac{1}{2}-\frac{1}{2} \frac{1}{2}-\frac{3}{2}-\frac{1}{2} \quad \frac{1}{2} \quad \frac{3}{2}-\frac{1}{2}-\frac{1}{2}-\frac{3}{2}-\frac{1}{2}-\frac{1}{2}-\frac{3}{2}-\frac{5}{2} \cdot \cdots$

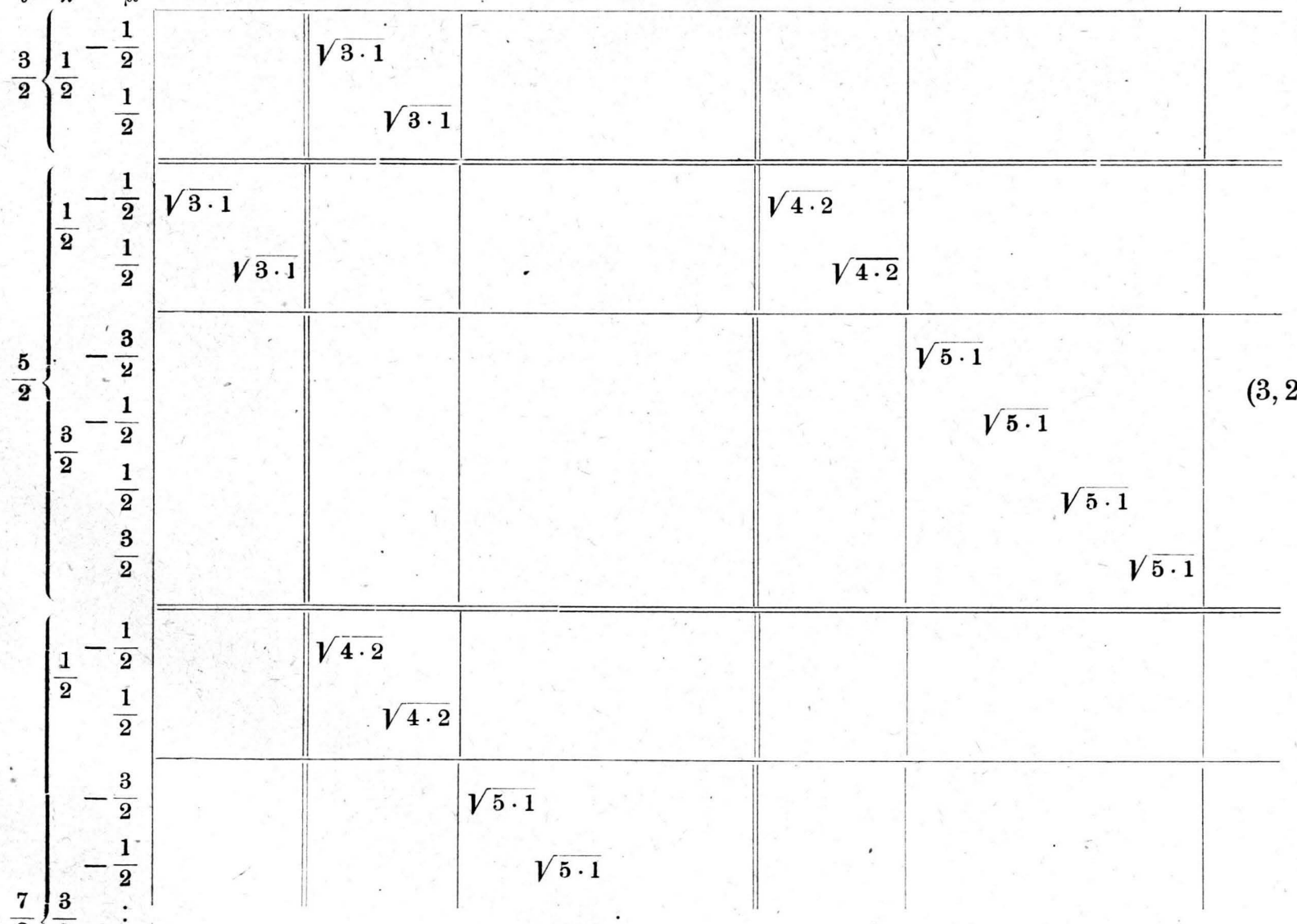


Auch die übrigen Matrizen lassen sich nach den angegebenen Formeln ohne weiteres hinschreiben. Die Äquivalenz mit der früheren Darstellung, in der offenbar alles eindeutig ist, sorgt dafür, daß die Faktoren $(3,23)$ mit den Werten $x=0$ und $1 / 2$, für die sie unbestimmt würden, wegen Verschwindens der betreffenden $\delta$-Faktoren nicht auftreten.

Was die Eigenwerte betrifft, so sind die von I, $\mathrm{K},:^{4}, \mathbf{x}^{4}$ und von den drei $\mathbf{M}_{k}$-Komponenten schon angegeben. Die der übrigen Matrizen erhält man ohne Bezugnahme auf die frühere Darstellung, indem man geeignete Zyklen nach Art von (4) in 2 bildet. So bilden z. B. $x_{1}$ und $x_{2}$ mit $M_{3}$ den Zyklus

$\left[\varkappa_{1} \varkappa_{2}\right]=i \mathrm{M}_{3},\left[\mathrm{M}_{3} \varkappa_{1}\right]=i \varkappa_{2},\left[\varkappa_{2} \mathrm{M}_{3}\right]=i \varkappa_{1}$,

mit drei positiven Vorzeichen, woraus man sofort schließt, daß ihre Eigenwerte ganz oder halbganz sind. Die entsprechenden :-Komponenten bilden dagegen

$\left[\iota_{1} \iota_{2}\right]=-i \mathrm{M}_{3},\left[\mathrm{M}_{3} \iota_{1}\right]=i \iota_{2},\left[\iota_{2} \mathrm{M}_{3}\right]=i \iota_{1}$,

mit einem negativen Zeichen, woraus nach der Schlußweise von 2 folgt, daß sie kontinuierliche Spektren haben. Diese Folgerung entspricht derjenigen von $\mathrm{H}$ ill $1^{8}$ mit dem Unterschiede, daß, wie man sieht, $\iota^{4}$ mit diskretem und sogar wesentlich positivem Eigenwertspektrum gebraucht werden kann. Diese Matrix wird hiermit bei gegebenem I gerade zum Schlüsselelement [s. etwa die Matrix $(3,28)$ für K]. Die interessante Erscheinung, daß die Komponenten eines und desselben Viereroder Sechservektors ganz verschiedenen Spektralcharakter haben können, findet man in ${ }^{1}$ und besonders in ${ }^{9}$ genauer erörtert.

In ähnlicher Weise ist auf die kontinuierliche Natur der $\Pi_{k}$-Komponenten zu schließen. Sie lassen sich wieder mit je einer $\mathbf{M}_{k}$-Komponente $\mathrm{zu}$ Tripeln der Form $(3,30)$ zusammenfassen. Das negative Vorzeichen läßt sich heraustransformieren, wenn man alle $\Pi_{k}$ durch $i \Pi_{k}$ ersetzt. Danach entsprechen ihre V.-R. denen des Drehimpulses und lassen sich durch endliche Matrizen erfüllen. Auf diese Weise entstehen die wohlbekannten endlichen Darstellungen der Lorentz-Transformation. Aber die $\Pi_{k}$-Komponenten werden dabei rein imaginär. Dadurch erklärt sich das oft bemerkte Auftreten eines imaginären elektrischen Momentes beim Diracschen Elektron, wenn man durch Bildung von Kommutator-Relationen auf seinen Spin zu schließen versucht. Die invarianten
V.-R. von Dirac sind eben nicht Kommutator-, sondern Antikommutator-Relationen. Man kann bekanntlich mit den Operatoren von Dirac auch einen reellen Sechservektor bilden. Um aber ihre V.-R. in eine kommutator-ähnliche, invariante Form zu bringen, muß man einen invarianten Faktor einschalten, d.h. jedes $a b-b a$ durch ein $a \cdot \operatorname{Inv} \cdot b-b \cdot \operatorname{Inv} \cdot a$ ersetzen ${ }^{17}$. Unsere PoissonKlammern erfordern demgegenüber, wenn man sie getreu übersetzen will, reine KommutatorRelationen. Das Verlangen, diese mit reellen Größen zu erfüllen, war der ursprüngliche Antrieb für die Entwicklung der vorliegenden Darstellungstheorie.

\section{We ll eng le i chung und Hamilton-Funktion}

Nach der Schlußweise von 1, welche der in der Relativitätsmechanik üblichen bis auf die Trennung von Impuls und Geschwindigkeit entspricht, hat man den Faktor $m$ in der Invarianten $g_{k} u^{k}=-m c$, wo $g_{k}$ der Impuls mit Einschluß des Vektorpotentials und $u^{k}$ die Vierergeschwindigkeit ist, als Ruhmasse aufzufassen. Die Invarianz würde nicht ausschließen, daß $m$ noch von $\mathrm{I}$ und $\mathrm{K}$ abhinge, doch wiesen schon die Betrachtungen im Schlußabschnitt von 1 darauf hin, $m$ als Konstante $\left(m_{0}\right)$ zu betrachten, und auch der Versuch einer Deutung der Mesonenmassen in 2 ging von dieser Voraussetzung aus. Wir wollen der Einfachheit halber auch hier daran festhalten. Da die Vierergeschwindigkeit $u^{k}$ als $\iota^{\mathrm{k}} / \sqrt{\mathrm{I}^{2}+\mathrm{K}^{2}}$ aufzufassen ist - vgl. 1 oder $2-$, haben wir für die kräftefreie Bewegung

$$
\iota_{k} p^{k}=-m_{0} c \sqrt{\mathrm{I}^{2}+\overline{K^{2}}} .
$$

Die $p^{k}$ sind die Komponenten des Impulses, $c p^{4}$ ist die Energie. Mit ihrem Ersatz durch die entsprechenden Differentialoperatoren kommt man, wie üblich, zur Wellengleichung, durch Auflösung nach $p^{4}$ zur Hamilton-Funktion.

Was die erstere betrifft, so ist zu beachten, daß die $\iota^{k}, k=1,2,3$, keineswegs antikommutativ, noch mit $:^{4}$ vertauschbar sind, daher die Wellengleichung nicht die Gordon-Schrödingersche Gleichung nach sich zieht, was gewöhnlich im Anschluß an Dirac für wesentlich gehalten wird.

17 Bezüglich dieser anscheinend weniger beachteten Verhältnisse sei auf W. Wessel, Z. Physik 96, 520 [1935], hingewiesen. 
Da wir hier nicht die Wellengleichung postulieren, sondern die V.-R., die nun wenigstens im Falle $\mathbf{I}=0$ durch Bopp eine so klare Begründung aus der klassischen Theorie erfahren haben, scheint uns dieser Verzicht nicht bedenklich. Ubrigens hat auch schon Bhabha ${ }^{18}$ nicht darauf Rücksicht genommen. Aus dem gleichen Grunde begegnet, soviel wir sehen, die Einführung äußerer Felder durch den Übergang von $p^{k}$ zu $g^{k}$ nicht der bekannten Schwierigkeit von $\mathrm{Pauli}$ und $\mathrm{F}$ i e r z ${ }^{19}$. Er führt in der klassischen Theorie auf Grund der angenommenen P.-K., wie in 1 gezeigt. wurde, auf die richtigen Bewegungsgleichungen. und die Umdeutung der P.-K. in V.-R. bringt jedenfalls keine Symmetrieschwierigkeiten mit sich.

Die Gl. $(4,1)$ erscheint also als physikalisch wohlbegründet. Wir haben auch schon in 2 gezeigt, wie man das Massenproblem damit auf halbklassischem Wege recht anschaulich diskutieren kann. Ihre strenge Behandlung führt freilich auf schwierige mathematische Probleme, in deren Lösung wir noch keine mitteilenswerten Fortschritte machen konnten. Zwei mehr physikalische Fragen, die dabei noch auftreten, dürften aber zu beantworten sein.

Die erste betrifft die quantentheoretische Definition der Quadratwurzel in $(4,1)$. Man könnte daran denken, sie durch

$$
\left(\begin{array}{lr}
\mathrm{I} & \mathrm{K} \\
\mathrm{K} & -\mathrm{I}
\end{array}\right)
$$

zu ersetzen, da I und $\mathrm{K}$ kommutieren, doch verliert man damit die positive Definitheit der Energie, die im übrigen durch den wesentlich positiven Charakter von ' $^{4}$ gewährleistet ist. Der richtige Weg ist wohl der, von einer K-Darstellung auszugehen, in der die Wurzel wohldefiniert ist, und sie von dort in die ${ }^{4}$-Darstellung überzuführen, in der sich die Matrizen am bequemsten angeben lassen. Die Bestimmung der unitären Matrix, d.h. der Eigenfunktionen $\Phi_{\iota}(\mathrm{K})$, die $\mathrm{K}$ diagonal machen, ist nur eire Frage der Transformationstechnik, denn $\mathbf{K}$ ist in den Darstellungen erster Art unmittelbar durch einen Differentialoperator 2. Ordnung gegeben.*

18 H. J. Bhabha, Rev. mod. Physics 17, 200 [1945].

19 M. F i e r z u. W. P a u i , Proc. Roy. Soc. [London], Ser. A 173, 211 [1939].
Die zweite Frage betrifft die Auflösung von $(4,1)$ nach dem Energieparameter bzw. die Bestimmung der Hamilton-Funktion. Es sei in $(4,1)$, unter Bezugnahme auf ein bestimmtes Koordinatensystem, $p^{4}=m_{0} c \cdot \eta$ gesetzt und die Gleichung in die Form gebracht

$$
\eta \iota^{4}-\mathrm{r}^{\mathrm{c}}=0 \text {. }
$$

$:^{4}$ und $\mathrm{I}^{2}$ sind nicht vertauschbar. Gefragt ist nach den Eigenwerten von $\eta$. Soll man $\eta$ gleich dem hermiteschen Realteil von $\left(!^{4}\right)^{-1} \Upsilon$ setzen, oder was sonst? Der gewohnten quantenmechanischen Schlußweise ordnet man sich wohl am besten folgendermaßen ein. Man wählte eine Darstellung, in der $\imath^{4}$ diagonal ist mit Elementen $\imath_{k}^{4}$. In dieser Form ist die Matrix bei uns gerade gegeben. Die Elemente von $\Upsilon \mathrm{Y}$ in dieser Darstellung seien $\mathrm{Y}_{i k}$; durch unsere Darstellung ist dafür gesorgt, daß sie hermitesch sind: $\Upsilon_{k i}^{*}=\Upsilon_{i k}$. Die Säkulargleichung lautet dann

$$
\left|\begin{array}{ccc}
\mathrm{r}_{11}-\eta \iota_{1}^{4} & \mathrm{r}_{12} & \mathrm{r}_{13} \ldots \\
\mathrm{r}_{21} & \mathrm{r}_{22}-\eta \iota_{2}^{4} & \mathrm{r}_{23} \ldots \\
\ldots & &
\end{array}\right|=0 .
$$

Sie hat nicht die Standardform, nimmt sie aber sogleich an, wenn man jede Zeile und Kolonne durch die Wurzel aus dem zugehörigen Eigen. wert von $:^{4}$ dividiert:

$$
\left|\frac{\Upsilon_{i k}}{\left(\iota_{i}^{4} \iota_{k}^{4}\right)^{1 / 2}}-\eta \delta_{i k}\right|=0 .
$$

Indem man dies als $\left|H_{i k}-\eta_{i k}\right|=0$ interpretiert, erhält man die Hamilton-Funktion $\mathrm{H}_{i k}$ in der Form

$$
\mathrm{H}=\left(\iota^{4}\right)^{-1 / 2} \Upsilon\left(\iota^{4}\right)^{-1 / 2} .
$$

Der Operator ist offenbar hermitesch und wohldefiniert, falls kein Eigenwert von $:^{4}$ verschwindet oder negativ ist, was für unsere sämtlichen Darstellungen zutrifft. Für $p_{1}=p_{2}=p_{3}=0^{*}$ erhält man insbesondere den Operator der Ruhmasse. Es wäre sehr interessant, zu wissen, ob er in dieser Form schon diskrete Eigenwerte hat, oder ob vielleicht (was wir eher erwarten möchten) unscharfe Eigenwerte erst durch entsprechende Übergangswahrscheinlichkeiten ausgezeichnet sind.

* Anm. b. d. Korr. Wurde inzwischen direkt im Anschluß an $(3,22)$ erledigt. 